\title{
The Presence of Venous Gas Does Not Affect the Prognosis in Emphysematous Cystitis
}

\author{
Yuki Yoshimatsu ${ }^{1}$, Tomoko Takai ${ }^{2}$, Yasuhisa $\mathrm{Abe}^{2}$ and Toshimasa Nakagawa ${ }^{3}$
}

\begin{abstract}
Objective Emphysematous cystitis (EC) has a high mortality rate compared with urinary tract infection without emphysema. However, its prognostic factors have yet to be determined. The presence of venous gas is suspected to be a rare, adverse prognostic factor of EC. However, all four previously reported cases improved. We hypothesized that venous gas is not an adverse prognostic factor of EC and aimed to assess the effect of venous gas on the EC prognosis.

Methods Medical records were reviewed retrospectively.

Patients The patients diagnosed with EC at Yodogawa Christian Hospital between April 2004 and September 2014 were included.

Results Venous gas was present in 15 of 23 patients with EC. There was no significant difference in the background or clinical presentation between patients with or without venous gas. All patients with venous gas survived without invasive measures, whereas $50 \%$ of patients without venous gas died.

Conclusion There was no marked difference in the mortality rate due to EC between the patients with and without venous gas. Venous gas may be a more common and less worrying finding in EC than assumed. It does not reflect the severity of infection, and air embolisms have not been reported so far. Venous gas may not affect the prognosis. This may be due to the differences in the mechanism of venous gas production. Gas in EC may develop due to glucose fermentation and intravesical pressurization, in contrast to the necrotizing infection seen in other emphysematous infections. This is the first study to assess the effect of venous gas on EC prognosis.
\end{abstract}

Key words: emphysematous cystitis, venous gas, emphysematous pyelonephritis, urinary tract infection, air embolism

(Intern Med 56: 637-640, 2017)

(DOI: 10.2169/internalmedicine.56.7601)

\section{Introduction}

Emphysematous cystitis (EC) is a unique, complicated urinary tract infection, where gas is present in the bladder wall. Major risk factors include diabetes mellitus (DM), urinary tract obstruction, old age, and female sex. Patients typically present with common symptoms such as fever, chills, abdominal pain, nausea, and vomiting. The causative bacteria are similar to those of simple urinary infections such as Escherichia coli and Klebsiella pneumoniae. The clinical presentation is similar to that of cystitis or nephritis without emphysema $(1,2)$.

Gas in the bladder wall is thought to be the result of bacteria fermenting high levels of tissue glucose, and the subsequent escalation of carbon dioxide production and accumulation. The presence of gas along the bladder wall on abdominal X-ray or computed tomography (CT) is necessary for a diagnosis. The standard treatment consists of systemic administration of antibiotics and vesical depressurization with a urinary catheter $(1,2)$. The diagnosis and treatment differ from non-emphysematous urinary tract infections.

The majority of cases respond well to conservative measures. Refractory cases or cases managed inappropriately

${ }^{1}$ Department of Respiratory Medicine, Yodogawa Christian Hospital, Japan, ${ }^{2}$ Department of Diabetes and Endocrinology, Yodogawa Christian Hospital, Japan and ${ }^{3}$ Clinical Laboratory, Yodogawa Christian Hospital, Japan

Received for publication April 19, 2016; Accepted for publication June 26, 2016

Correspondence to Dr. Yuki Yoshimatsu, yukitsukihana0105@gmail.com 


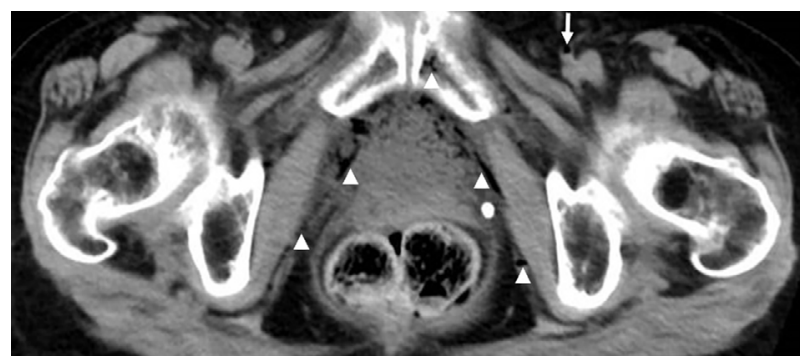

Figure. Computed tomography image of a patient with emphysematous cystitis. Gas is seen in the femoral vein (arrow) and branches of the internal iliac vein (arrowheads).

may progress to emphysematous pyelonephritis and/or require surgical intervention, such as urinary stenting and nephrectomy (3). Mortality is reported to be $7 \%$ with EC alone, $14 \%$ when complicated with other emphysematous infections, and over 50\% when complicated with emphysematous pyelonephritis (2).

Recently, with the widespread use of CT scans, the diagnosis rate of EC is increasing. In particular, the diagnosis rate of venous gas has inarguably increased with CT scans compared with abdominal X-rays. Venous gas in EC is assumed to develop as glucose in the urinary tract is fermented, and the carbon dioxide produced migrates to the veins due to urinary tract damage from bacteria and elevated intravesical pressure (4). In emphysematous pyelonephritis, gas advancing out of the kidneys is recognized as an adverse prognostic factor (5). In EC, the frequency of intravascular gas and its effect on prognosis has not been determined. So far, there have only been four reported cases of EC with venous gas. Each case improved with treatment $(4,6-8)$.

To the clinician, intravascular gas in the presence of an infection is an alarming finding, suggesting the involvement of gas-forming bacteria or bacteremia. The presence of gas in a normally gasless area generally calls for immediate attention. However, we suspected that venous gas might not affect the prognosis of EC. Therefore, we examined the relationship between EC and venous gas in cases diagnosed in our hospital. To our knowledge, this is the first study to analyze the effect of venous gas on the prognosis of EC.

\section{Materials and Methods}

The medical records of patients diagnosed with EC at Yodogawa Christian Hospital between April 2004 and September 2014 were reviewed retrospectively. The diagnostic criteria of EC have yet to be established. We included cases diagnosed clinically, using a urinalysis and radiologic imaging. Venous gas is diagnosed when gas is detected within a vein. On X-ray, the intrapelvic veins cannot be seen, and venous gas is also undetectable. Therefore, only patients who underwent abdominal CT were included. We divided the patients into two groups based on the presence of venous gas and compared the patient background, clinical course, treat- ment, and outcome. This study was approved by the institutional ethics committee of Yodogawa Christian Hospital.

\section{Results}

There were a total of 23 cases of EC, in 12 men and 11 women. The mean age was 76.4 years old. Common comorbidities included neuropsychiatric conditions in 16 cases (dementia 8 cases, stroke 7, epilepsy 2, depression 2, schizophrenia 1, mental retardation 1, Parkinsonism 1), malignant tumor in 15 cases, and diabetes mellitus and urologic disorders in 10 cases. Venous gas was found in 15 cases. Gas was detected in the branches of the internal iliac vein in 14 cases, and in the femoral vein in 3 cases (Figure). There was only 1 case who developed septic shock, and 1 case with disseminated intravascular coagulation.

The patients were divided into two groups depending on the presence of venous gas (Table). There were no significant differences in the patient background or clinical presentation between the two groups, except for the overall mortality rate. EC patients without venous gas had a mortality rate of $50 \%$, whereas all patients with venous gas survived. There was no marked difference in the mortality rate due to EC in either group.

In the four patients who died, the selected treatment did not differ markedly from that in those who survived. EC had shown improvement in all patients, and the causes of death were status epilepticus, rectal cancer, gastric cancer, and suffocation. The frequency of cancer or other complications in EC patients with venous gas did not differ significantly from those without gas.

Treatment with antibiotics and urinary catheterization tended to be selected more often in EC patients with venous gas. Broad-spectrum antibiotics were selected in some cases with venous gas, out of concern for the presence of gasproducing microbiomes and severe conditions. Some patients also underwent multiple CT scans to check for venous gas improvement. Venous gas was not present on these follow-up CT scans.

\section{Discussion}

Patient background, including age, sex, and comorbidities, did not differ markedly from previous reports. In this study, nearly $70 \%$ had an underlying neuropsychiatric condition. In these patients, abnormal urination habit, neurogenic bladder dysfunction, and medication (such as anticholinergic agents) may have contributed to the risk of increased intravesical pressure and urinary infection. Urinary retention due to benign prostatic hypertrophy was also seen quite commonly. There were $17.4 \%$ asymptomatic cases, highlighting the importance of a proactive diagnostic approach, especially in elderly patients.

The Table shows that the overall mortality rates of EC patients were $50 \%$ and $0 \%$, depending on the presence of venous gas. In contrast to previous assumptions, the mortality 
Table. Patient Background, Clinical Presentation, Isolated Pathogens, and Outcome of EC Patients.

\begin{tabular}{|c|c|c|c|c|}
\hline & & Total & With venous gas & No venous gas \\
\hline \multicolumn{2}{|l|}{ Total cases } & 23 & 15 & 8 \\
\hline \multicolumn{2}{|l|}{ Male sex } & $12(52.2 \%)$ & $8(53.3 \%)$ & $4(50.0 \%)$ \\
\hline \multicolumn{2}{|l|}{ Mean age } & 76.3 & 76.9 & 75.4 \\
\hline \multicolumn{5}{|l|}{ Comorbidity } \\
\hline \multicolumn{2}{|c|}{ Neuropsychiatric condition } & $16(69.6 \%)$ & $11(73.3 \%)$ & $5(62.5 \%)$ \\
\hline \multicolumn{2}{|c|}{ Malignant tumor } & $15(65.2 \%)$ & $9(60.0 \%)$ & $6(75.0 \%)$ \\
\hline \multicolumn{2}{|c|}{ Diabetes mellitus } & $10(43.5 \%)$ & $7(46.7 \%)$ & $3(37.5 \%)$ \\
\hline \multicolumn{2}{|c|}{ Urologic disorders } & $10(43.5 \%)$ & $8(53.3 \%)$ & $2(25.0 \%)$ \\
\hline \multicolumn{5}{|c|}{ Symptom } \\
\hline \multirow{4}{*}{ Urologic } & Hematuria & $12(52.2 \%)$ & $8(53.3 \%)$ & $4(50.0 \%)$ \\
\hline & Urinary retention & $11(47.8 \%)$ & $8(53.3 \%)$ & $3(37.5 \%)$ \\
\hline & Incontinence & $6(26.1 \%)$ & $4(26.7 \%)$ & $2(25.0 \%)$ \\
\hline & Frequent urination & $4(17.4 \%)$ & $3(20.0 \%)$ & $1(12.5 \%)$ \\
\hline \multirow{2}{*}{ Systematic } & Fever & $8(34.8 \%)$ & $6(40.0 \%)$ & $2(25.0 \%)$ \\
\hline & Fatigue & $3(13.0 \%)$ & $2(13.3 \%)$ & $1(12.5 \%)$ \\
\hline \multicolumn{2}{|c|}{ Asymptomatic } & $4(17.4 \%)$ & $1(6.7 \%)$ & $3(37.5 \%)$ \\
\hline \multicolumn{5}{|c|}{ Culture positivity } \\
\hline \multicolumn{2}{|c|}{ Blood } & $4 / 14(28.6 \%)$ & $3 / 9(33.3 \%)$ & $1 / 5(20.0 \%)$ \\
\hline \multicolumn{2}{|l|}{ Urine } & $15 / 17(88.2 \%)$ & $10 / 11(90.0 \%)$ & $5 / 6(83.3 \%)$ \\
\hline \multicolumn{5}{|l|}{ Pathogen } \\
\hline \multicolumn{2}{|c|}{ Escherichia coli } & $11(47.8 \%)$ & $7(46.7 \%)$ & $4(50.0 \%)$ \\
\hline \multicolumn{2}{|c|}{ Klebsiella pneumoniae } & $3(13.0 \%)$ & $2(13.3 \%)$ & $1(12.5 \%)$ \\
\hline \multicolumn{2}{|c|}{ Citrobacter koseri } & $1(4.3 \%)$ & $1(6.7 \%)$ & $0(0 \%)$ \\
\hline \multicolumn{5}{|l|}{ Treatment } \\
\hline \multicolumn{2}{|c|}{ Antibiotic and urinary catheter } & $14(60.9 \%)$ & $11(73.3 \%)$ & $3(37.5 \%)$ \\
\hline \multicolumn{2}{|c|}{ Antibiotic only } & $4(17.4 \%)$ & $1(6.7 \%)$ & $3(37.5 \%)$ \\
\hline \multicolumn{2}{|c|}{ None } & $5(21.7 \%)$ & $3(20.0 \%)$ & $2(25.0 \%)$ \\
\hline \multicolumn{2}{|l|}{ Septic shock } & $1(4.3 \%)$ & $1(6.7 \%)$ & $0(0 \%)$ \\
\hline \multicolumn{2}{|c|}{$\begin{array}{l}\text { Disseminated intravascular } \\
\text { coagulation }\end{array}$} & $1(4.3 \%)$ & $1(6.7 \%)$ & $0(0 \%)$ \\
\hline \multicolumn{2}{|c|}{ Overall death } & $4(17.4 \%)$ & $0(0 \%)$ & $4(50 \%)$ \\
\hline
\end{tabular}

rate was clearly higher in EC without venous gas than with gas. Previous reports of EC with venous gas assumed it was an adverse prognostic factor without any previous data of similar cases. All four reported cases of EC with venous gas have recovered. This is the first study to report multiple cases of EC with venous gas. Therefore, we hypothesized that in cases of EC, the presence of venous gas may not be an adverse prognostic factor. There were no significant differences in the comorbidities or other clinical findings between the two groups. There was only 1 case who developed septic shock, and 1 case with disseminated intravascular coagulation, both in the group with venous gas. It was not possible to determine whether or not this finding was of significance in the present study.

In general, clinicians are cautious when patients present with venous gas. This is probably because we encounter venous gas more often in severe infections, such as intestinal ischemia or renal abscess. Furthermore, gas spreading out from the urinary tract in emphysematous pyelonephritis has been reported to be an adverse prognostic factor (5). It is therefore conceivable to also extend this concept to EC with venous gas. However, the presence of gas has not been reported to be an adverse prognostic factor in EC (9). This discrepancy between the effect of venous gas in EC and other infections may be due to differences in the mechanism of gas production.

In emphysematous nephritis, tissue ischemia due to severe inflammation causes necrotizing infections $(10,11)$. In intestinal ischemia, severe infection due to proliferation of anaerobic bacteria, causing tissue necrosis and gas production, may result in venous gas (12). The difference between EC and these infections may be that our EC patients presumably did not develop necrotizing infections. Additionally, because urinary retention is the primary cause of EC and venous gas, it is possible to directly relieve the cause by urinary catheterization. The fact that pressure and gas can be released in a simple and quick way may have also contributed to the low mortality rate of EC with venous gas compared to other infections with venous gas.

Some studies have suggested that air embolisms may contribute to the high mortality rate of emphysematous urinary tract infections (8). In previous reports of EC with venous gas, due to suspected air embolism, one patient underwent a lung perfusion scan (7), whereas another underwent hyperbaric oxygen therapy as a preventative measure (6). Of the 15 cases in the present study, there were no cases of air em- 
bolism related to venous gas. Venous gas was shown to disappear over time with multiple CTs. This may be partially due to early effective depressurization by urinary catheterization. To date, there is no evidence of EC causing air embolisms. Therefore, broad-spectrum antibiotics or invasive measures may not be necessary to treat EC and venous gas alone, unless indicated by other conditions.

The present study shows that venous gas is a relatively common complication in EC and that it is not an adverse prognostic factor. The mortality rate due to EC did not differ markedly between the patients with and without venous gas. Our opinion is that this may be due to the absence of tissue necrosis, and also due to early vesical depressurization and drainage by urinary catheterization. No complications due to venous gas, such as air embolism, were observed. This study was a retrospective study with a limited number of cases. EC is generally seen in elderly patients, and its high mortality rate demands careful attention. A larger study is therefore necessary before general recommendations can be made.

The authors state that they have no Conflict of Interest (COI).

\section{References}

1. Amano M, Shimizu T. Emphysematous cystitis: a review of the literature. Intern Med 53: 79-82, 2014.

2. Thomas AA, Lane BR, Thomas AZ, Remer EM, Campbell SC, Schoskes DA. Emphysematous cystitis: a review of 135 cases.
BJU Int 100: 17-20, 2007.

3. Evanoff FV, Thompson CS, Foley R, Weinman EJ. Spectrum of gas within kidney; emphysematous pyelonephritis and emphysematous pyelitis. Am J Med 83: 149-154, 1987.

4. Nozu T. Emphysematous cystitis with air bubbles in the inferior vena cava. Int J Urol 15: 947, 2008.

5. Huang JJ, Tseng CC. Emphysematous pyelonephritis: clinicoradiological classification, management, prognosis, and pathogenesis. Arch Intern Med 160: 797-805, 2000.

6. McCabe JB, Mc-Ginn Merritt W, Olsson D, Wright V, Camporesi EM. Emphysematous cystitis: rapid resolution of symptoms with hyperbaric treatment: a case report. Undersea Hyperb Med 31: 281-284, 2004.

7. Karashima E, Ejima J, Nakamura H, Koike A, Kaneko T, Ohmura I. Emphysematous cystitis with venous bubbles. Intern Med 44: 590-592, 2005.

8. Yokokawa R, Tsuka H, Muranaka K. Emphysematous cystitis with air bubbles in the vena cava. Nihon Hinyokika Gakkai Zasshi 105: 22-25, 2014 (in Japanese, Abstract in English).

9. $\mathrm{Lu} \mathrm{YC}$, Chiang BJ, Pong YH, et al. Predictors of failure of conservative treatment among patients with emphysematous pyelonephritis. BMC Infect Dis 14: 418, 2014.

10. Uruc F, Yuksel OH, Sahin A, Urkmez A, Yildrim C, Verit A. Emphysematous pyelonephritis: our experience in managing these cases. Can Urol Assoc J 9: E480-E483, 2015.

11. James PN, Chalmers AG, Fowler RC. Ultrasound demonstration of gas in hepatic veins. Clin Radiol 40: 429-430, 1989.

12. Nelson AL, Millington TM, Sahani D, et al. Hepatic portal venous gas: the ABCs of management. Arch Surg 144: 575-581, 2009.

The Internal Medicine is an Open Access article distributed under the Creative Commons Attribution-NonCommercial-NoDerivatives 4.0 International License. To view the details of this license, please visit (https://creativecommons.org/licenses/ by-nc-nd/4.0/).

(C) 2017 The Japanese Society of Internal Medicine http://www.naika.or.jp/imonline/index.html 